\title{
NUMERICAL STUDY OF ELASTIC WAVE PROPAGATION IN A LAYERED BAR
}

\author{
R. Kolman ${ }^{*}$, A. Berezovski* ${ }^{* *}$, M. Berezovski ${ }^{* * *}$
}

\begin{abstract}
In this paper, we numerically study elastic wave propagation in a heterogeneous bar. Different compositions of the layered bar are assumed as well as number of layers and different materials. The effect of bar composition on stress distribution is analyzed. For numerical simulations, the finite element method and the finite volume method are used in the sense of explicit time integration. Numerical computations show the strong influence of layer compositions on the dispersion of a pulse and stress distribution in a heterogeneous bar. The numerical prediction of stress wave propagation in heterogeneous bars helps for the setting of suitable experimental specimens made by additive technologies for studies of wave propagation in such materials.
\end{abstract}

Keywords: elastic wave propagation, dispersion, heterogeneous solid, layered bad, finite element method, finite volume method

\section{Introduction}

Elastic wave propagation in a heterogeneous solid bar as a one-dimensional case is the typical test problem for models of composite materials, see (Nayfeh, 1995; Chakraborty and Gopalakrishnan, 2003). The numerical modelling is necessary because macroscopic properties of composite materials are strongly influenced by the properties of their constituents. The macroscopic properties are usually determined by a homogenization, which yields effective stresses and strains acting on materials, e.g. see (Srivastava, 2015).

Layered periodic materials represent the simplest possible pattern of composites from the theoretical point of view. Their modelling as periodic structures also has a rich history, see the book of Brillouin (1946). Several geometrical problems of elastic wave propagation in layered media have been studied in book of Nayfeh (1995). Constitutive models of effective properties for such materials are still under development using either ensemble averaging, see (Willis, 2009), or integration over unit cell, see (Nemat-Nasser and Srivastava, 2011). These models work well for sufficiently long waves. For short waves, it is worth to use numerical simulations for the analysis of interaction between layers and waves taking into account reflected and transmitted waves.

Moreover, numerical simulation of wave propagation even in a homogeneous solid bar under shock loading is under discussion so far both in the context of finite volume methods, see (Ketcheson et al., 2013) and finite element methods, see (Idesman et al., 2014; Kolman et al., 2016a, 2017). This is why two different numerical methods - finite element method and finite volume method - are applied in the paper for the simulation of a pulse propagation in a slender heterogeneous solid bar. The dispersion behaviours of the finite element method have been studied in work of (Kolman et al., 2013, 2016b). The objective of the paper is to demonstrate the influence of the composition of alternating layers on the dispersion of a pulse and to compare results of simulation obtained by two different numerical methods. Further, numerical simulations help to understanding of wave propagation processes in heterogeneous bodies and setting of material composition of specimens for experimental studies.

Ing. Radek Kolman, Ph.D.: Institute of Thermomechanics, v.v.i., The Czech Academy of Sciences, Dolejškova 1402/5; 182 00, Prague 8; CZ, kolman@it.cas.cz

** Prof. Arkadi Berezovski: School of Science, Tallinn University of Technology, 21 Akadeemia Rd.; 12618, Tallinn; Estonia, Arkadi.Berezovski@cs.ioc.ee

**** Prof. Mihhail Berezovski: Department of Mathematics, Embry-Riddle Aeronautical University, 600 S. Clyde Morris Boulevard, Daytona Beach; Florida 32114-3990, USA, mihhail.berezovski@erau.edu 


\section{Problem definition}

We interest in elastic wave propagation in a bar with the length $L$, the constant cross-section and piecewise constant distribution of material parameters as an ideal layered bar. The motion is assumed being onedimensional and considered within the linear theory of elastodynamics, e.g., (Graff, 1975). The balance equation of linear momentum takes the form

$$
\rho \frac{\partial v}{\partial t}-\frac{\partial \sigma}{\partial x}=0
$$

where $\rho$ is the matter density, $v$ is the particle velocity, $v=\partial u / \partial t, u(x, t)$ is the particle displacement, $\sigma$ is the one-dimensional Cauchy stress, $t$ is the time and $x$ is the position. In the linear elasticity the Cauchy stress obeys the Hooke law, $\sigma=E \varepsilon$, where $E$ is the Young modulus and $\varepsilon$ is the one-dimensional infinitesimal strain, $\varepsilon=\partial u / \partial x$. The wave speed in a bar is given for one-dimensional case by $c(x)=$ $\sqrt{E(x) / \rho(x)}$, thus it yields $\sigma=\rho c^{2} \varepsilon$. The strain and velocity are related to the compatibility condition

$$
\frac{\partial \varepsilon}{\partial t}=\frac{\partial v}{\partial x}
$$

In terms of the displacement, the balance of linear momentum is represented in the form of the linear wave equation

$$
\frac{\partial^{2} u}{\partial t^{2}}=c^{2} \frac{\partial^{2} u}{\partial x^{2}}
$$

It should be noted that the material parameters $\rho, E$ and $c$ are distinct in different parts of the bar. However, they keep constant values for each computational cell/element in numerical methods for the bar with piecewise constant distribution of material parameters such as Young modulus $E$ and matter density $\rho$.

\section{Numerical procedures}

In this paper, we compare results obtained by the finite element method (FEM) and the finite volume method (FVM) in case of explicit approaches of these methods. In FEM, the classical explicit time integration by the central difference method in time and the linear finite element method in space with the lumped mass matrix is employed, for details see (Hughes, 1983). In FVM, the thermodynamic consistent explicit scheme presented in the book by Berezovski et al. (2008) is used for elastic wave simulation in a layered bar. The both methods are conditionally stable, thus one has to choose the time step size with respect to the stability limit, see the book of Hughes (1983). In this paper, we select the time step size as a minimum value of local time step sizes over all cells: $\Delta t=\min \left(h / c_{e}\right)$, where $h$ is the grid size and $c_{e}=\sqrt{E_{e} / \rho_{e}}$ is the wave speed corresponding to $e$-th cell.

\subsection{Numerical results}

We assume a layered bar with two different material compositions with three materials. The compositions of material along the bar are depicted in Figs.1. In the modelling setting, there are following material parameters as material 1: $\rho_{1}=1 \mathrm{~kg} / \mathrm{m}^{3}, c_{1}=1 \mathrm{~m} / \mathrm{s}$, with corresponding $E_{1}=1 \mathrm{~Pa}$; material 2: $\rho_{2}=0.6 \mathrm{~kg} / \mathrm{m}^{3}, c_{2}=0.96 \mathrm{~m} / \mathrm{s}$, with corresponding $E_{2}=0.55296 \mathrm{~Pa}$; material 3: $\rho_{3}=0.8 \mathrm{~kg} / \mathrm{m}^{3}$, $c_{3}=0.98 \mathrm{~m} / \mathrm{s}$, with corresponding $E_{3}=0.76832 \mathrm{~Pa}$.

The length of a bar is assumed $L=1 \mathrm{~m}$. For this purpose, the interval $0 \leq x \leq L$ is divided into $N=1500$ cells/elements of the same size. The thickness of each layer is equal to 30 space elements (grid cells). The
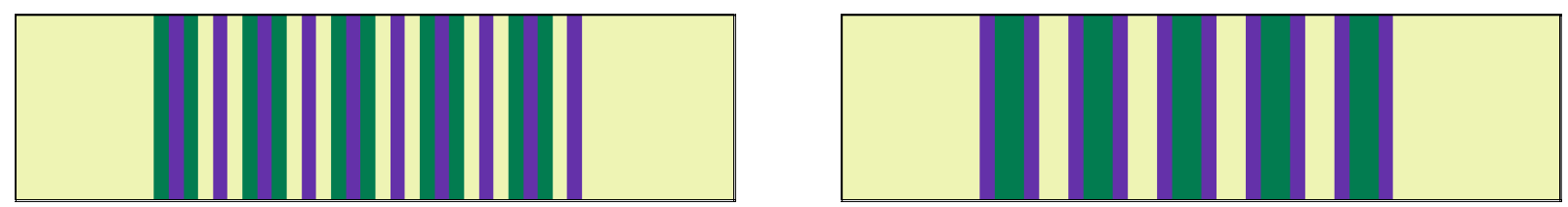

Fig. 1: Distributions of three-phase periodic layers. Variant no. 1 on the left. Variant no. 2 on the right. Yellow domain - material 1, green domain - material 2, blue domain - material 3. 


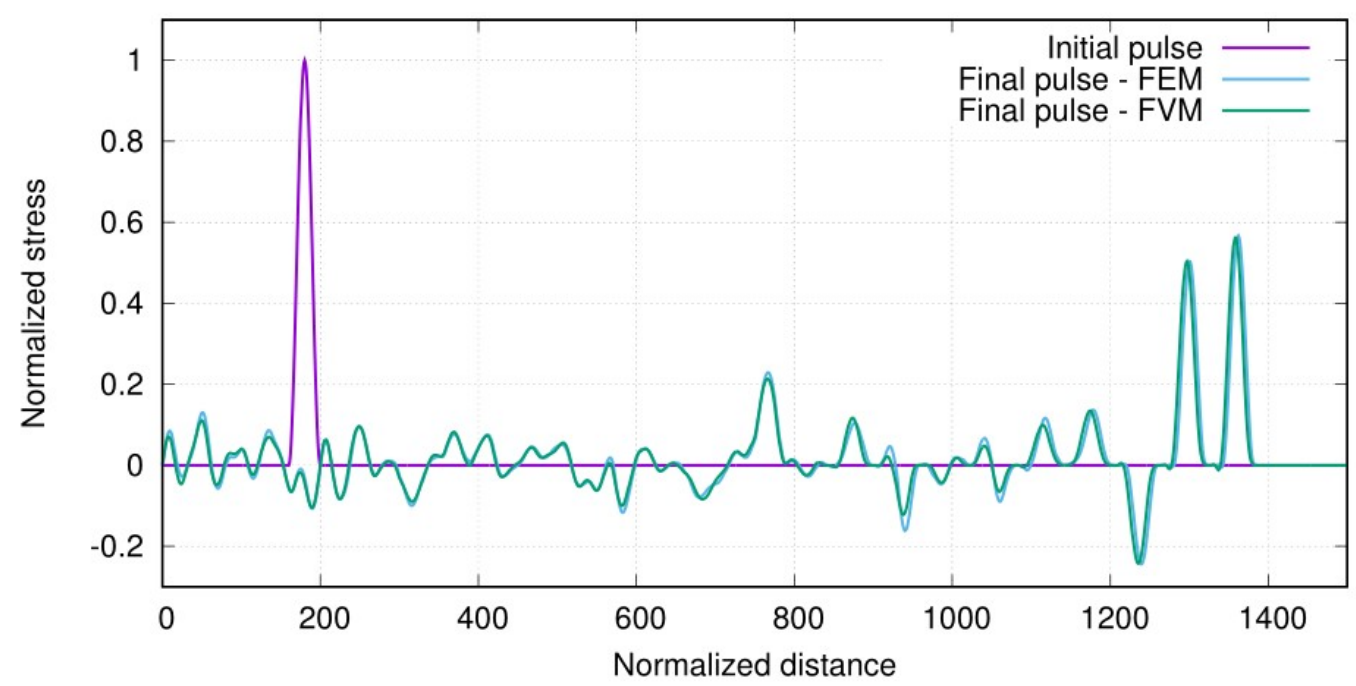

Fig. 2: Stress distribution of three-phase periodic layered bar - Variant 1.

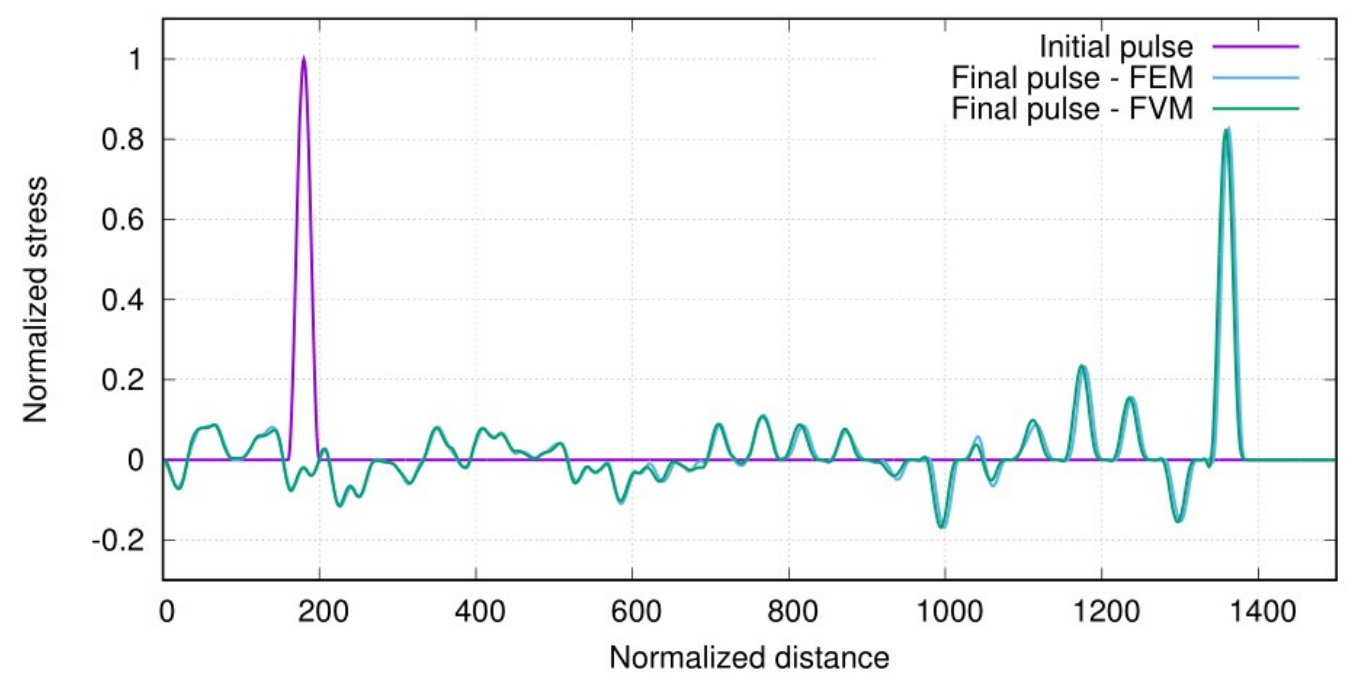

Fig. 3: Stress distribution of three-phase periodic layered bar - Variant 2.

alternating layers (thick left and right boundary layers marked by the yellow colour) are placed starting from the 300-th grid cell until the 1200-th grid cell. In this setting we study wave propagation through layers of the bar and results are not influenced by reflected waves from boundaries of the bar.

The bar is loading by the applied tension traction on the left side with the time history given as $\sigma(0, t)=$ $\frac{1}{2}(1+\cos (\pi(t-20) / 20))$ for normalized (dimensionless) stress. The bar is fixed on the right side, thus the displacement is zero; $u(L, t)=0$. The computed distributions of the stress for the final time corresponding to 1400 time step sizes are shown in Figs. 2 and 3 for the both assumed variants of material compositions of the layered bar.

We present the results of simulations of elastic wave propagation in heterogeneous bars with different compositions. In Figs. 2 and 3, one can see the results of elastic wave propagation through the layered bars of variant 1 and variant 2 defined in Fig. 1. Here the initial pulse is depicted. At the first, the obtained results of stress distributions in the bar by FEM and FVM are in principle very close. Only small differences can be observed, which are given with different prescribing of boundary conditions for each numerical method. Further, based on simulations, the stress distribution is strongly depended on geometrical and material compositions of layers. Mainly the maximum of stress distribution can be controlled by optimization of layers. i.e. their width and material. This is the principle of application of layered materials in impact and ballistic problems, see (Rosenberg and Dekel, 2016). 


\section{Conclusions}

Composite materials as layered materials are broadly applied in industry and real applications because of their excellent designable characteristics - mechanical behaviour and lower mass weight. The recent advancement in additive manufacturing eased the fabrication of complex structures with a very wide range of materials. We have presented, the numerical results of elastic wave propagation in the heterogeneous bar, as our an initial work, and results will help for the setting of experimental specimens made by additive manufacturing. The both used numerical strategies - the finite element method and the finite volume method - produce in principle the same stress distribution in presented one-dimensional numerical tests. Based on numerical simulations, we are able to set optimal material compositions of the heterogeneous bar with respect to space distributions of materials and also to the time duration of applied loading/pulse. In the future, we will focus on multi-dimensional modelling of wave propagation in complex heterogeneous and anisotropic media, as functionally graded materials.

\section{Acknowledgements}

The work of R. K. was supported by the grant projects with No. 17-22615S of the Czech Science Foundation (CSF) within institutional support RVO:61388998. The work of R. K. and A. B. was supported by the Centre of Excellence for Nonlinear Dynamic Behaviour of Advanced Materials in Engineering CZ.02.1.01/0.0/0.0/15_003/0000493 (Excellent Research Teams) in the framework of Operational Programme Research, Development and Education.

\section{References}

Berezovski, A., Engelbrecht, J. and Maugin, G.A. (2008) Numerical Simulation of Waves and Fronts in Inhomogeneous Solids, World Scientific.

Brillouin, L. (1946) Wave Propagation in Periodic Structures: Electric Filters and Crystal Lattices, McGraw-Hill Book Company.

Chakraborty, A. and Gopalakrishnan, S. (2003) Various numerical techniques for analysis of longitudinal wave propagation in inhomogeneous one-dimensional waveguides, Acta Mechanica, Vol 162, No.1-4, pp 1-27.

Graff, K.F. (1975), Wave Motion in Elastic Solids, Clarendon Press.

Hughes, T.J.R. (2008) The Finite Element Method: Linear Static and Dynamic Finite Element Analysis, Dover Civil and Mechanical Engineering, Dover Publications.

Idesman, A., Pham, D., Foley, J.R. and Schmidt, M. (2014) Accurate solutions of wave propagation problems under impact loading by the standard, spectral and isogeometric high-order finite elements. Comparative study of accuracy of different space-discretization techniques, Finite Elements in Analysis and Design, Vol 88, pp 67-89.

Ketcheson, D.I., Parsani, M. and LeVeque, R.J. (2013) High-order wave propagation algorithms for hyperbolic systems, SIAM Journal on Scientific Computing, Vol 35, No. 1, pp A351-A377.

Kolman, R., Plešek, J., Okrouhlík, M. and Gabriel, D. (2013) Grid dispersion analysis of plane square biquadratic serendipity finite elements in transient elastodynamics, International Journal for Numerical Methods in Engineering, Vol 96, No. 1, p 1-28.

Kolman, R., Cho, S.S. and Park, K.C. (2016) Efficient implementation of an explicit partitioned shear and longitudinal wave propagation algorithm, International Journal for Numerical Methods in Engineering, Vol 107, No. 7, pp 543-579.

Kolman, R., Plešek, J., Červ, J., Okrouhlík, M. and Pařík, P. (2016) Temporal-spatial dispersion and stability analysis of finite element method in explicit elastodynamics, International Journal for Numerical Methods in Engineering, Vol. 106, No. 2, p 113-128.

Kolman, R., Okrouhlík, M., Berezovski, A., Gabriel, D., Kopačka, J. and Plešek, J. (2017) B-spline based finite element method in one-dimensional discontinuous elastic wave propagation, Applied Mathematical Modelling, Vol 46, pp 382-395.

Nayfeh, A.N. (1995) Wave Propagation in Layered Anisotropic media, North-Holland.

Nemat-Nasser, S. and Srivastava, A. (2011) Overall dynamic constitutive relations of layered elastic composites, Journal of the Mechanics and Physics of Solids, Vol 59, No.10, pp 1953-1965.

Rosenberg, Z. and Dekel, E. (2016) Terminal Ballistics, 2nd ed., Springer.

Srivastava, A. (2015) Elastic metamaterials and dynamic homogenization: a review, International Journal of Smart and Nano Materials, Vol 6, No.1, pp 41-60.

Willis, J.R. (2009) Exact effective relations for dynamics of a laminated body, Mechanics of Materials, Vol 41, No.4, pp 385-393. 